\title{
Comparison of cable models for time domain simulations
}

\author{
W.Z. El-Khatib*, J. Holboell*, T.W. Rasmussen* S. Vogel* \\ *Technical University of Denmark, Kgs. Lyngby, Denmark-wzel@elektro.dtu.dk
}

\begin{abstract}
This paper will look at dc cable transient modelling issues for voltage source converter (VSC) based high voltage direct current (HVDC) transmission systems, which is getting evermore admired for large-scale offshore wind power integration. A simple mathematical $\pi$ equivalent cable model is presented and explained. The model is compared to the detailed cable models with different degrees of accuracy found in PSCAD/EMTDC. The models are analyzed and tested using simulations of dc fault conditions and energization. These studies are helpful for the protection system design of large-scale renewable energy power systems to realize a reliable multi-terminal dc transmission system for the future.
\end{abstract}

\section{Introduction}

HIGH voltage direct current (HVDC) is a competitive transmission option for large-scale renewable energy integration to distant utility grids [1], [2]. Investigation of errors in the simulation of HVDC cables have in the past years become a necessity due to the increased deployment of HVDC around the world. Today with further usage of HVDC due to the VSC and a large increase in HVDC connected WPP's, this problem is again of relevance, how to carry out the simulation and design of the power transmission systems for HVDC links, including long submarine HVDC cables. Obviously, a new cable model with a higher accuracy, especially ever more popular multi-terminal HVDC, is required. Detailed mathematically calculated $\pi$-circuits equivalent are known to give relatively good simulation results for cable. However when long distance cables are to be modelled the number of $\pi$-circuit equivalents increases drastically. Thus the computation time in most time domain programs becomes unacceptable. A solution for a simple and accurate time domain cable model has yet to be found. As a well known fact, the problem is due to that the cable and the cable screens etc [3] and [4]. are inherently frequency dependent, and it is difficult to simulate the frequency dependent characteristics of a cabling system directly in time domain, for instance, by using the cable model of the EMTP and EMTDC program [5]. In the PSCAD/EMTDC program the two cable models, namely the Bergeron model and the frequency dependent model with a constant transformation, are included. A third model is also given called "frequency dependent model with frequency dependent transformation”. This work will compare detailed mathematically calculated Pi-equivalent models to the PSCAD/EMTDC cable models. Frequency sweeps will be performed for all models to investigate and analyse the various cable model performances and frequency dependency capabilities. Furthermore fault analysis will be conducted as well, to give an overview of the frequency ranges the EMTDC models are valid in and the consequences of frequency dependent deviations. The main finding of the work is to indicate if the PSCAD/EMTDC model can be used for transient studies and identify the model limitations if any.

A conclusive part of the work covers further discussion of the results, a summary of the main findings and proposals for future work on the field.

\section{Modeling}

In this section an example of mathematical cable model is discussed and explained. Furthermore the methods used to obtain the PSCAD/EMTDC cable model values are clarified.

\subsection{Mathematical model}

The skin-effect exists for electromagnetic wave to travel in a conductive media, and is dependent on only the characteristics of the material and the propagating wave frequency. As a convenient measure to evaluate the skin-effects, the "skin-depth" concept is used. One can think of the wave as having essentially disappeared after penetrating a few values of the skin-depth. Normally, the skin depth, $\delta$, in a homogenous conductive media is calculated by the following formula,

$$
\delta=\sqrt{\frac{1}{\pi f \sigma \mu}}
$$

Where $f$ is frequency, $\sigma$ is the conductivity, and $\mu$ is the permeability. The skin-depths for the configuration of HVDC cable deep in seawater has to be found individually for the cable main conductor, the cable lead sheath, the cable armour steel tapes the cable armour steel wires and for the seawater [6].

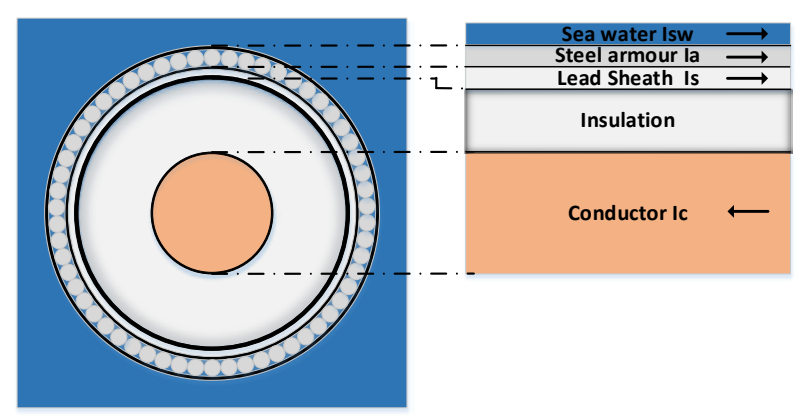

Figure 1 Cable cross-section 
According to an ordinary design of a HVDC cable transmission system, the lead sheath and the steel armour of the cable are always connected together and earthed to the local earthing system at two terminations. The dc voltage is applied on the insulation between the main conductor and lead sheath. As illustrated as in Fig. 1 , the transmitted dc and low frequency current shall go through the main conductor and returns through the three different ways in parallel: 1) the lead sheath, 2) the armour and 3) the seawater. The reason is explained that if all of the currents in the conductors of returning path operate in the same direction, the electric and magnetic interaction within the returning path conductors through the insulations has to be very small. Only the internal impedances of the conductors of three returning path are dominant, and shall therefore be included in the cable model. Acceding to the basic concepts of cable model above, a $\pi$-circuit section per unit length for the low frequency cable model may be established as shown in Fig. 2

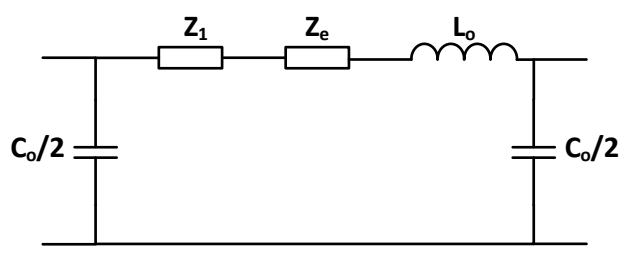

Figure 2 Matmathical $\pi$-model

Where $Z_{1}$ is the internal impedance of the main conductor of cable and $\mathrm{Ze}$ is the total impedance of the current returning path. For the main conductor the method is to use a R-L circuit of some type to approach the frequency characteristics of the impedance $Z_{1}$ for which has an inductive characteristics. With a laddershape circuit, the boundary condition is that the impedance $Z_{1}$ at dc reduces to the parallel combination of all resistors, $\mathrm{R} 1$ through $\mathrm{Rn}$, and equals to the dc resistance of the cable main conductor.

$$
Z_{1}=Z_{2}\left(f_{2}\right) / / Z_{3}\left(f_{3}\right) \ldots . / / Z_{n}\left(f_{n}\right)
$$

The calculation of Ze is formulated as equation.

$$
Z e=Z e_{2} / / Z e_{3} / / Z e_{4} / / Z e_{5}
$$

Where the parameters are $\mathrm{Ze}_{2}$ for the internal impedance of lead sheath, $\mathrm{Ze}_{3}$ for the internal impedance of the armour steel sheath, $\mathrm{Ze}_{4}$ for the internal impedance of the armour wires and $\mathrm{Ze}_{5}$ for the internal impedance of seawater. The $\mathrm{C}_{0}$ and $\mathrm{L}_{0}$ are the capacitance and inductance over the main insulation between the main conductor and lead sheath, respectively.

At low frequency, $Z_{1}$ is equal to the dc resistance of the main conductor. At high frequency the skin effect must be included. The formula in [7] may calculate $Z_{1}$ in both low and high frequencies. That is,

$$
\begin{aligned}
& \left.Z_{1}=\frac{\rho_{1}}{\pi r_{1}^{2}}+\frac{\mu_{0} \mu_{r 1}}{8 \pi}\right\} r_{1} \leq 2 \delta_{1} \\
& \left.\begin{array}{l}
Z_{1}=\frac{1}{2 r_{1}} \sqrt{\frac{\mu_{0} \mu_{r 1} \rho_{1}}{\pi}} \cdot \sqrt{f} \\
+\frac{1}{4 \pi r_{1}} \sqrt{\frac{\mu_{0} \mu_{r 1} \rho_{1}}{\pi}} \cdot \frac{1}{\sqrt{f}}
\end{array}\right\} r_{1} \leq 2 \delta_{1}
\end{aligned}
$$

Where $\delta_{1}$ is the skin depth of the main conductor. The unit is $O h m / m$ for $R_{1}$ and $H / m$ for $L_{1}$. The internal impedance of the lead sheath is given by the formula[5].

$$
\begin{gathered}
Z_{2,3}=\frac{\rho_{2}}{2 \pi \sqrt{r_{2,4} r_{3,5} \cdot \delta_{2,3}}} \\
\text { For } r_{2,4} \geq T_{2,3} \leq \delta_{2,3}
\end{gathered}
$$

Where $\delta_{2,3}$ is the skin depth of the lead sheath, the sheath thickness $T_{2}=r_{3}-r_{2}, T_{3}=r_{5}-r_{4}$ and the impedance unit is $\mathrm{Ohm} / \mathrm{m}$. Owing to the neglecting of the shielding effects of the armour wire ring, the total impedance, $Z_{4}$, shall be only the internal impedances of all wires. For each armour wire, the internal impedance, z4, including skin-effects may be calculated using the same method as for the impedance, $Z_{1}$, applying the formula (4) and (5). Then the total impedance is.

$$
Z_{4}=\frac{1}{n w} z_{4}
$$

Where nw is the total number of the armour wires. The formula for the self impedance of the seawater $Z_{5}$ of the immersed cable has been worked out by $\mathrm{E}$ Vance [8].

\subsection{PSCAD/EMTDC model}

In PSCAD/EMTDC we find four models that are used for cable modelling.

1) $\pi$-Models: This model consist of the well known lumped $\mathrm{R}$ and $\mathrm{L}$, but an equally divided $\mathrm{C}$ at both ends. The concept of $\pi$-equivalent model is also used in a distributed system. The distributed $\pi$ model results in high-order functions which will take long time to be solved or simulated [10].

2) Bergeron Model: The Bergeron model is a development of the simple $\pi$-model. It is a constant frequency model based on travelling wave theory. The cable is considered to be lossless infinite number of $\pi$ sections. It accurately represents the distributed $L$ and $C$, but resistance $\mathrm{R}$ is added as a series lumped component. Thus the resistance is not represented in a distributed manner. Furthermore all parasitic components are not included in the model as it the case in the $\pi$-equivalent model. As a result of this the model has none or minimum frequency dependency. 
It is accurate at a specified frequency and is suitable for studies where the specified frequency is important [11]. However, for harmonics and electromagnetic compatibility (EMC) study, some frequency response information will be missed with this and the above simple $\pi$-model.

3) Travelling Wave Model in the modal domain (Frequency Dependent in Mode): As the name indicates, frequency dependent (FD) models are models that have frequency dependent cable parameters. This is achieved by using the cable's propagation and admittance matrices. When compared with the Bergeron models, the use of the frequency domain increases the results accuracy. In FD-models all calculations are performed in the frequency domain and the solutions converted to time domain by the using transformations such as Fourier-transform or Z-transform. The frequency dependent model in mode is achieved with a constant transformation matrix [10]. The frequency dependent model represents the frequency dependence of all parameters (not just at the specified frequency as in the Bergeron model). This model uses modal techniques to solve the cable constants and assumes a constant transformation. It is therefore only accurate for systems of ideally transposed conductors (or 2 conductor horizontal configurations) or single conductors.

4) Travelling Wave Model 2 (Frequency Dependent in Phase): The frequency dependent phase model also represents the frequency dependence of all parameters as in the above model. However, the problem of a frequency dependent transformation matrix will be overcome by formulating the model directly in the phase domain (without diagonalization). This is the most accurate model [10] since it includes frequency dependence of internal transformation matrices.

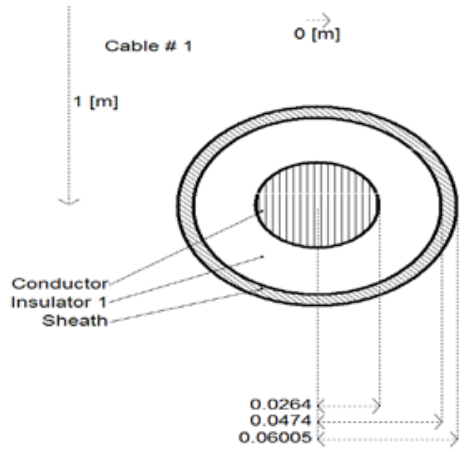

Figure 3 PSCAD coaxial cable model EMTDC configuration (for dc underground dc positive and negative cables).

Both the Bergeron and the frequency dependent models in PSCAD/EMTDC are applied as comparison, which includes all the layers: copper core, insulation, armour and sheath, shown in Fig. 3.
Most simulation software cannot model segmented and stranded conductors, as it only allows the modelling of solid and hollow conductors, which due to skin effect are not used for cables with large cross-sectional areas. A conductor's DC resistance is calculated as shown in (8), where $l$ is the conductor length, $\rho$ the material's electrical resistivity and A the conductor's crosssectional area. The cross-section of a segmented conductor as given in the datasheet is not equal to $\pi \mathrm{r}^{2}$, where $r$ is the conductor radius. This difference is used to correct the resistivity as shown in (8), where $\rho$ is the material's resistivity and $\rho$ ' the corrected resistivity.

$$
R_{D C}=l \frac{\rho}{A}=l \frac{\rho^{\prime}}{\pi r^{2}} \Leftrightarrow \rho^{\prime}=\rho \frac{\pi r^{2}}{A}
$$

The DC resistance of the main conductor is given by the manufactory. However this resistance can be adjusted to the proper temperature level using the following equation.

$$
R_{D C_{-} \text {cor }}=R_{D C}\left[1+\alpha_{20}(\theta-20)\right]
$$

Where $R_{D C}$ is the given conductor resistance, $\alpha_{20}$ is the metal temperature coefficient at $20^{\circ}$ per kelvin and $\Theta$ is the nominal operating temperature.

\section{Simulation results}

In this section the simulation results of the two models are compared and the results are explained. The mathematical model is compared to the three models available in PSCAD/EMTDC the Bergeron, the frequency dependent phase and mode models.

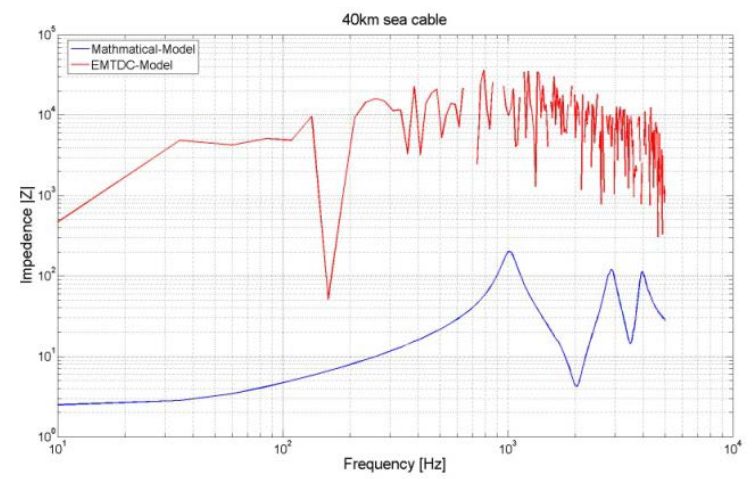

Figure 4 Bergeron Model compared to mathematical $\pi$ model $40 \mathrm{~km}$ cable.

As mentioned earlier the Bergeron model doesn't include any parasitic components. Hence the model has none or minimum frequency dependency. This is clearly seen from Fig. 4 where the EMTDC results seem more as a calculation errors compared to the mathematical model. This is clearly due to the change in frequency that the model isn't able to compensate for. Initially the cable is inductive, however with increasing frequency the skin and proximity effects will start to influence the impedance. Consequently making the cable capacitance 
dominate, nevertheless this behaviour isn't seen for the EMTDC Bergeron model.

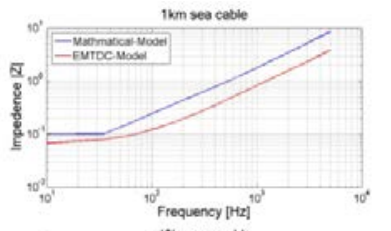
$40 \mathrm{~km}$ sea cable

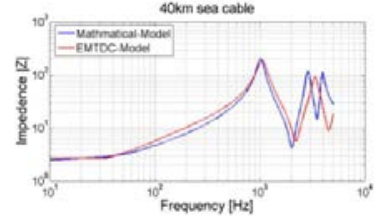

Figure 5 Frequency responds for Frequency Dependent (Mode) Model compared to mathematical $\pi$-model for different cable lengths.

When comparing to the Frequency Dependent (Mode) Model, the two models behaves similarly, due to the inclusion of frequency dependency. However it is clear that deviations will occur at higher frequencies.

Increasing the cable length will increase the effect of the parasitic components. Hence the resonances are moved to lower frequencies Fig. 5.
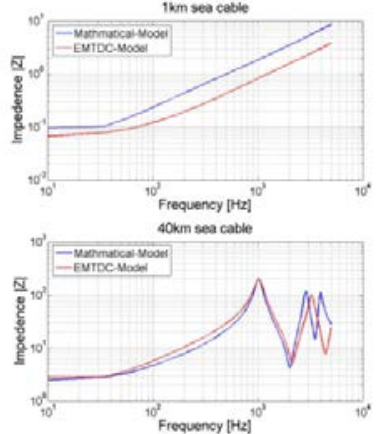

Figure 6 Frequency responds for Frequency Dependent (Phase) Model compared to mathematical $\pi$-model for different cable lengths.

The Frequency Dependent (Phase) Model is according to PSCAD/EMTDC the best model to use when investigating high frequent behaviour since it includes frequency dependence of internal transformation matrices. Nonetheless when comparing the two FD models Fig. 5 and 6 they appear alike however the resonances are slightly shifted in frequency.

The mathematical $\pi$-model simulation results are close enough to the accurate cable model as shown in Fig. 7 which zoomed to show some minor difference between the two simulation results. This is due to the various frequency components in the overcurrent. However it clear that when looking at the low frequency oscillations the two models behaves almost identically.

On the other hand when simulating an energization using the two models Fig. 8, the travelling wave behaves similar in both models. Nonetheless the in the mathematical $\pi$-model there is a high frequency oscillations of $4 \mathrm{kHz}$ that can't be found in the EMTDC model.

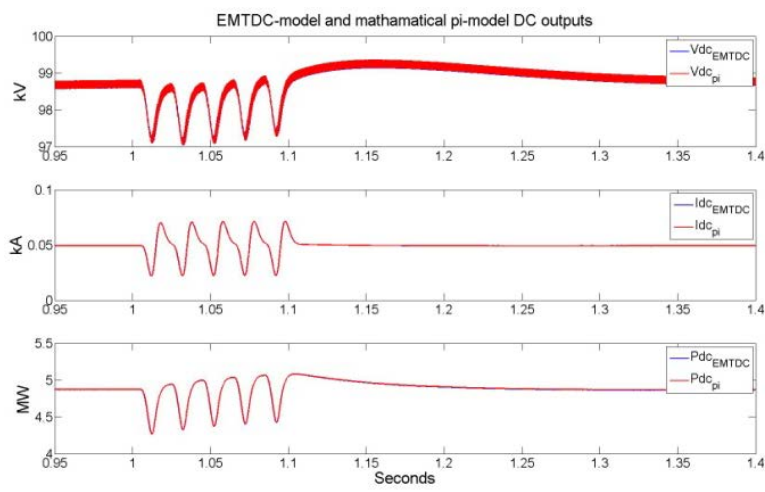

Figure 7 DC-line outputs for rectifier phase arm fault for both models

This can be explained from the lower plots in Fig. 6 where it is noticed that the EMTDC model has greater damping in the higher frequencies compared to mathematical $\pi$-model.
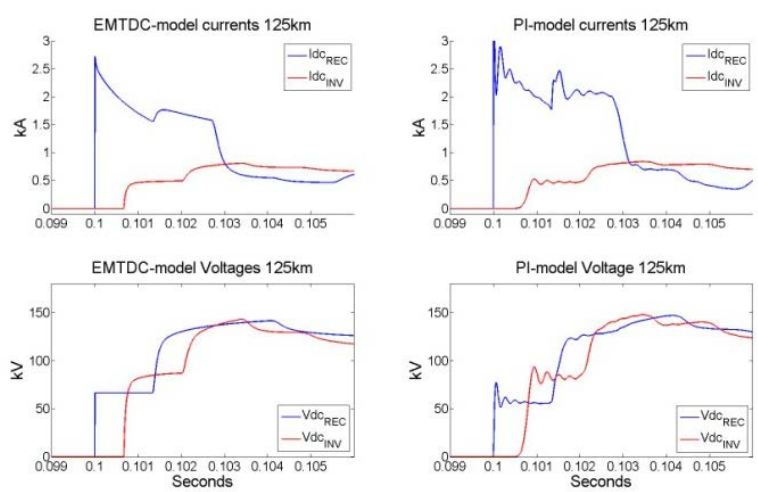

Figure 8 Energization simulation for both models

\section{Conclusion}

VSC-HVDC transmission systems are a new area primed by the potential development of multi-terminal offshore HVDC. In this paper, dc cable modelling issues are analyzed for transient dc fault energization analysis. Various dc cable models are summarized following analysis of some cable events to compare the models for accurate and efficient simulations. Simulation results show that for low-frequency faults, the mathematical $\pi$ model is adequate for simulation and protection relay design. Hence standards for dc system fault protection are urgently required for dc network operation. However when considering high frequency transients the models differ in the high frequencies. Hence resulting in oscillations in the mathematical $\pi$-model. Thus more analysis is needed to investigate the exactness of the cable model.

\section{References}

[1] P. Bresesti, W. L. Kling, R. L. Hendriks, and R. Vailati, "HVDC connection of offshore 
wind farms to the transmission system," IEEE Trans. Energy Convers., vol. 22, no. 1, pp. 3743, Mar. 2007.

[2] N. Flourentzou, V. G. Agelidis, and G. D. Demetriades, "VSC-based HVDC power transmission systems: an overview," IEEE Trans. Power Electron., vol. 24, no. 3, pp. 592602, Mar. 2009.

[3] Alan Budner, "Introduction of Frequency-Dependent Line Parameters into an Electromagnetic Transients Program", IEEE Transactions on

Power Apparatus and Systems, Vol. PAS-89, No. 1, January 1970

[4] H. M. J. De Silva, A. M. Gole, J. E. Nordstrom, L. M. Wedepohl, "Robust Passivity Enforcement Scheme for Time-Domain Simulation of Multi-Conductor Transmission Lines and Cables", IEEE Transactions on Power Delivery, Vol. 25, Issue 2, April 2010

[5] CIGRE Joint Working Group 21/22.01, "Comparison of High Voltage Overhead Lines and Underground Cables - Report and Guidelines", 1996

[6] R. Bartnikas, K. D. Srivastava "Power and Communication Cables: Theory and Applications", IEEE Press: Power Engineering Series, 2000

[7] K C Chen and K M Damrau, “Accuracy of approximate transmission line formulas for overhead wires", IEEE Trans. on Electroma. Compati., Vol. 31, No. 4, Nov. 1989, pp 396-397

[8] E F Vance, "Coupling to shielded cables", John Wiley \& Sons, Inc. 1978, pp 75-76 and Table 3.3

[9] C S Yen, Z Fazarinc, and R L Wheeler, “ Timedomain skin effect model for transient analysis of lossy transmission”, Proceeding of the IEEE, Vol. 70, No. 7, July 1982.

[10] B. Gustavsen, G. Irwin, R. Mangelrod, D. Brandt, and K. Kent, "Transmission line models for the simulation of interaction phenomena between parallel ac and dc overhead lines,” Int. Conf. on Power Sys. Transients, Budapest, Hungary, 20-24 Jun. 1999.

[11] PSCAD/EMTDC online help, version 4.2.1, Manitoba, Canada. 